\title{
ESSENTIALLY CLOSED RADICAL CLASSES
}

\author{
N. V. LOI \\ (Received 19 May 1982) \\ Communicated by R. Lidl
}

\begin{abstract}
The main goal of this paper is to describe radical classes closed under essential extensions. It turns out that such classes are precisely the homomorphically closed scmisimple classes, and hence a radical class is essentially closed if and only if it is subdirectly closed. Moreover, a class is closed under homomorphic images, direct sums and essential extensions if and only if it is an essentially closed radical class. Also radical classes are investigated which are closed under Dorroh essential extensions only, such a radical class $\mathbf{R}$ consists of idempotent rings provided that $\mathbf{R}$ does not contain the ring of integers, meanwhile all the other radicals satisfy this requirement. A description of (hereditary and) Dorroh essentially closed radicals is given in Theorem 4 .
\end{abstract}

1980 Mathematics subject classification (Amer. Math. Soc.): 16 A 21.

\section{Preliminaries}

We shall work in the variety of all associative rings. Radical and semisimple classes will mean such classes in the sense of Kurosh and Amitsur and for the fundamentals of radical theory we refer to [6]. We shall make use of the upper radical operator $थ \mathcal{L}$ and semisimple operator $\delta$ acting on a class $\mathbf{C}$ of rings and defined by

$$
\begin{aligned}
& \text { थ } \mathbf{C}=\{A: A \text { has no nonzero homomorphic image in } \mathbf{C}\}, \\
& \Im \mathbf{C}=\{A: A \text { has no nonzero ideal in } \mathbf{C}\}
\end{aligned}
$$

respectively.

A class $\mathbf{C}$ is said to be hereditary if $I \triangleleft A \in \mathbf{C}$ implies $I \in \mathbf{C}$, and $\mathbf{C}$ is strictly hereditary, if for any subring $B$ of $A \in \mathbf{C}$ it follows $B \in \mathbf{C}$. A radical $\mathbf{R}$ consisting

Copyright Australian Mathematical Society 1983 
of idempotent rings will be referred to as a weakly subidempotent radical. A hereditarily weakly subidempotent radical is called subidempotent radical.

In our investigations the well-known notions of direct product (that is complete direct sum), direct sum (that is discrete direct sum) and subdirect sum will often occur. A class $\mathbf{C}$ is said to be closed under direct products (direct sums or subdirect sums) if any direct products (direct sum or subdirect sum respectively) of C-rings is again in $\mathbf{C}$. We say that $\mathbf{C}$ is closed under extensions or extensionally closed, if $B \in \mathbf{C}, A / B \in \mathbf{C}$ imply $A \in \mathbf{C}$. A class $\mathbf{C}$ is closed under essential extensions (or essentialiy closed), if $I \in \mathbf{C}$ implies $A \in \mathbf{C}$ for any essential (that is large) ideal $I$ of $A$. A ring $A$ is an essential extension of its ideal $I$ if $I$ is essential in $A$. If $A$ has also a unit element, in this case we say that $A$ is a unital essential extension of $I$.

In what follows $Z$ will stand for the ring of integers and $(n)$ will denote the ideal generated by $n \in Z$. If $n$ and $m$ are positive integers, then $\langle m, n\rangle$ denote their greatest common divisor.

\section{Radicals closed under essential extensions}

Let $A$ and $M$ be two rings. We shall say that $M$ is an $A$-algebra if

i) $M$ is an $A$-bimodule, and

ii) $a\left(m_{1} m_{2}\right)=\left(a m_{1}\right) m_{2} ;\left(m_{1} m_{2}\right) a=m_{1}\left(m_{2} a\right)$ hold for all $a \in A$ and $m_{1}, m_{2}$ $\in M$.

For an $A$-algebra $M$ let us consider the cartesian product $A \times M$ equipped with the operators

$$
\begin{aligned}
(a, m)+(b, n) & =(a+b, m+n), \\
(a, m) \cdot(b, n) & =(a b, a n+m b+m n) .
\end{aligned}
$$

It is easy to check that in this way we have built a ring $D(A, M)$ on the set $A \times M$ such that

a) $A \cong A_{0}=:\{(a, 0), a \in A\}$.

b) $M \cong M_{0}=:\{(0, m), m \in M\} \triangleleft D(A, M)$,

c) $D(A, M) / M_{0} \cong A$.

In view of (a), $D(A, M)$ can be considered as an $A$-algebra. An $A$-algebra $M$ will be said to be faithful, if

$$
\operatorname{Ann}_{A} M=:\{a \in A: a M=M a=0\}=0 .
$$

Lemma 1. If $I \triangleleft D(A, M)$ and $I \cap M_{0}=\{0\}$ then $I$ is isomorphic to an ideal of A. 
Proof. Since $I \triangleleft M_{0}=0$, using (c) it follows

$$
I \cong I /\left(I \cap M_{0}\right) \cong\left(I+M_{0}\right) / M_{0} \triangleleft D(A, M) / M_{0} \cong A .
$$

For any $A$-algebra $M$ let us consider the direct sum $N=\Sigma_{\lambda \in \Lambda} M_{\lambda}$ of infinitely many copies $M_{\lambda}$ of $M$. Clearly $N$ is an $A$-algebra in the obvious sense.

LeMmA 2. If $I \triangleleft D(A, N)$ and $I \cap N_{0}=0$ then

$$
I \subseteq\left\{(a, n): a \in \operatorname{Ann}_{A} M \text { and } n \in \operatorname{Ann}_{N} \bar{N}\right\} .
$$

Proof. Let $(a, n) \in I$ and $n=\Sigma_{\text {finite }} m_{\lambda_{i}}$. Since $I \cap N_{0}=0$, for any $(0, m) \in N_{0}$ we have

$$
a m+n m=m a+m n=0 .
$$

In particular, for $\mu \neq \lambda_{i}$ and for each element $m_{\mu} \in M_{\mu}$ such that for the corresponding element $m_{\mu}^{0}$ of $N_{0}$, we have $a m_{\mu}^{0}=m_{\mu}^{0} a=0$. Hence $a M=a M_{\mu}=0$ $=M_{\mu} a=M a$ holds, implying $a \in \mathrm{Ann}_{A} M$. Since $a \in \mathrm{Ann}_{A} M$, also $a \in$ $\operatorname{Ann}_{A} N$. Hence (*) yields $m n=n m=0$, that is $n \in \operatorname{Ann}_{A} N$.

The proof of the following assertion is straightforward.

LeMma 3. If $A_{\lambda}(\lambda \in \Lambda)$ are rings such that $\mathrm{Ann}_{A_{\lambda}} A_{\lambda}=0$ for each $\lambda \in \Lambda$, then their direct sum $\Sigma_{\lambda \in \Lambda} A_{\lambda}$ is an essential ideal in their direct product $\Pi_{\lambda \in \Lambda} A_{\lambda}$.

In the radical theory it is quite natural to demand that a semisimple class be essentially closed. This condition imposed on a semisimple class is in fact equivalent to the hereditariness of its radical class (see Armendariz [1]). It is not surprising that this condition imposed on radical classes, will be rather restrictive. Nevertheless, it is unexpected that the essentially closed radical classes are precisely the subdirectly closed ones, and the latter are known as the homomorphically closed semisimple classes. In the next theorem we give several characterizations of such classes.

THEOREM 1. For a class $\mathbf{C}$ of rings the following six conditions are equivalent:

i) $\mathbf{C}$ is an essentially closed radical class,

ii) $\mathbf{C}$ is closed under homomorphic images, direct sums and essential extensions,

iii) $\mathbf{C}$ is an essentially closed variety,

iv) $\mathrm{C}$ is an idempotent variety (that is, $\mathrm{C}$ is an extensionally closed variety),

v) $\mathbf{C}$ is a subdirectly closed radical class,

vi) $\mathbf{C}$ is a homomorphically closed semisimple class. 
Proof. The implication (i) $\Rightarrow$ (ii) is trivial. (ii) $\Rightarrow$ (iii): we must only prove that $\mathrm{C}$ is a variety. Let $B$ be an arbitrary nonzero subring of a ring $A \in \mathrm{C}$. Consider the unital essential extension $M$ of $A$, and the direct sum

$$
N=\sum_{i=1}^{\infty}\left(M_{i}: M_{i} \cong M, i=1,2, \ldots\right) .
$$

Since $\operatorname{Ann}_{B} M=0$ and $\operatorname{Ann}_{N} N=0$, for an ideal $I$ of the $\operatorname{ring} D(B, N)$ as given in the preliminaries, which satisfies $I \cap N_{0}=0$, Lemma 2 implies $I=0$. Hence $N_{0}$ is an essential ideal of $D(B, N)$. Using that $\mathbf{C}$ is closed under essential extensions and direct sums, $A \in \mathbf{C}$ implies $M \in \mathbf{C}, N_{0} \cong N \in \mathbf{C}$ and $D(B, N) \in$ C. Since $\mathbf{C}$ is homomorphically closed, property (c) yields $B \in \mathbf{C}$. Thus the class $\mathbf{C}$ is strictly hereditary.

Next let us consider unital essential extensions $M_{\lambda}$ of rings $A_{\lambda} \in C, \lambda \in \Lambda$. Since $\mathbf{C}$ is closed under direct sums, we have $\Sigma_{\lambda \in \Lambda} M_{\lambda} \in \mathbf{C}$ and an application of Lemma 3 yields $\Pi_{\lambda \in \Lambda} M_{\lambda} \in \mathrm{C}$. Taking into account that $\prod_{\lambda \in \Lambda} A_{\lambda} \subseteq \prod_{\lambda \in \Lambda} M_{\lambda}$ and that $\mathbf{C}$ is strictly hereditary as we have already proved-we get $\Pi_{\lambda \in \Lambda} A_{\lambda} \in \mathbf{C}$. Hence $\mathbf{C}$ is closed under direct products and strictly hereditary, thus $\mathbf{C}$ is a variety.

(iii) $\Rightarrow$ (iv). Since $\mathbf{C}$ is a variety, it is subdirectly closed and hereditary. Taking into account that $\mathbf{C}$ is also essentially closed an application of van Leeuwen's [3] Theorem $\mathbf{8}$ yields that $\mathbf{C}$ is closed under extensions. Thus $\mathbf{C}$ is an idempotent variety.

The equivalences of (iv), (v) and (vi) are well-known.

(vi) $\Rightarrow$ (i). It is well-known that a homomorphically closed semisimple class is a radical class (see Wiegandt [6] Theorem 32.1). Moreover if $\mathbf{C}$ is not the class of all rings, then every $\mathbf{C}$-ring is a subdirect sum of finite fields (see Stewart [4] Theorem 4.3 or Wiegandt [6]), hence it consists of semiprime rings. Suppose that $C$ is not the class of all rings, and let $B$ be an essential extension if its ideal $A \in \mathbf{C}$. By Birkhoff's Theorem there is a set $\left\{I_{\lambda}: \lambda \in \Lambda\right\}$ of ideals of $B$ such that each $B / I$ is subdirectly irreducible and $\bigcap_{\lambda \in \Lambda} I_{\lambda}=0$. Let us consider the set $\left\{I_{\mu}: A \not I_{\mu}, \mu\right.$ $\in \Lambda$ \}. Since $\left(\cap I_{\mu}\right) \cap A \subseteq \cap I_{\lambda}=0$ and $A$ is an essential ideal of $B$, it follows $\cap I_{\mu}=0$. Hence we can confine ourselves to the case $A \not I_{\lambda}$ for each $\lambda \in \Lambda$. Further, we have

$$
A^{\prime}=A /\left(I_{\lambda} \cap A\right) \cong\left(A+I_{\lambda}\right) / I_{\lambda} \triangleleft B / I_{\lambda}=B^{\prime} .
$$

Since $\mathrm{C}$ is a homomorphically closed class of semiprime rings every ideal of $A^{\prime}$ is also an ideal of $B^{\prime}$. Then the subdirect irreducibility of $B^{\prime}$ implies that of $A^{\prime}$. Hence by Stewart's result [4] Theorem $4.3 A^{\prime}$ is a finite field. Consequently $A^{\prime}=B^{\prime}$ for all subdirectly irreducible components of $B^{\prime}$ of $B$. Taking into consideration that $\mathbf{C}$ is subdirectly closed, it follows $B \in \mathbf{C}$ proving that $\mathbf{C}$ is essentially closed. 
Let us note that all homomorphically closed semisimple classes have been explicitly determined (see Gardner and Stewart [2]). There are countably many such classes and each of them is determined by a strictly hereditary finite set of finite fields (see Stewart [4] and our Theorem 4).

Let us recall that by Armendariz [1] (see also Wiegandt [6] Theorem 15.2) a semisimple class is essentially closed if and only if its radical class is hereditary. Hence an immediate consequence of Theorem 1 is the following

COROLlaRY. Let $\mathbf{C}$ be an essentially closed radical ciass, then the upper radical $9 \mathrm{C}$ is hereditary and the semisimple class $\delta \mathbf{C}$ is essentially closed.

\section{Dorroh essentially closed radicals}

We have seen in Section 2 that the requirement of being essentially closed is an extremely strong condition if it is imposed on a radical class. In this section we shall demand that the considered radical should be Dorroh essentially closed (the definition see below). This condition turns out to be a considerably weaker one inasmuch as every radical containing the ring of integers, does satisfy it, and it does not imply the hereditariness of the radical. Nevertheless, it is quite a restrictive condition, such a radical class. $\mathbf{R}$ consists of idempotent rings whenever $\mathbf{R}$ does not contain the ring of integers. If $Z \notin \mathbf{R}$ for a Dorroh essentially closed radical $\mathbf{R}$, then there is an integer $n>0$ with $n A=0$ for all $A \in \mathbf{R}$ (Proposition 3 ). A complete description of (hereditary and) Dorroh essentially closed radicals will be given in Theorem 4 .

Let $D(A)$ denote the Dorroh extension of a ring $A$ (that is $D(A)=D(Z, A)$ ). We say that a class $\mathbf{C}$ of rings is Dorroh essentially closed if $A \in \mathbf{C}$ implies $D(A) / I \in \mathrm{C}$ for every ideal $I$ of $A$ which is maximal with respect to the property $I \cap A=0$. This terminology is justified by the fact that in this case $A$ can be embedded as an essential ideal in $D(A) / I$. Since $D(A) / A \cong Z$ we get

Proposition 1. If $\mathbf{R}$ is a radical class containing $Z$. then $\mathbf{R}$ is Dorroh essentially closed.

For an arbitrary ring $A$ the characteristic char $A$ of $A$ is defined as

$$
\operatorname{char} A=\left\{\begin{array}{l}
\min (n: n A=0 \text { for some } n=1.2 \ldots) \text { if exists, } \\
0 \text { otherwise. }
\end{array}\right.
$$

Proposition 2. If $\mathbf{R}$ is a Dorroh essentially closed radical class and $Z \notin \mathbf{R}$, then char $A \neq 0$ for every $A \in \mathbf{R}$. 
Proof. If $A \in \mathbf{R}$, then also the direct sum $B=\sum_{i=1}^{\infty}\left(A_{i}: A_{i} \cong A\right)$ is in $\mathbf{R}$. Assume that $I$ is an ideal of the Dorroh extension $D(B)$ such that $I \cap B=0$ and $I$ is maximal with respect to this property. Since $D(B)=D(Z, B)$, Lemma 2 is applicable yielding

$$
I \subseteq\left\{(z, b): z \in \operatorname{Ann}_{Z} A \text { and } b \in \operatorname{Ann}_{B} B\right\} .
$$

Since $Z \notin \mathbf{R}$, it follows $D(B) \notin \mathbf{R}$ and hence $I \neq 0$. Using Lemma 1 we get that $I$ is isomorphic to an ideal of $Z$. Hence $I$ is generated by a single element $(z, b)$ such that $z \neq 0$. Since $I \cap B=0$,

$$
(0, z c+b c)=(z, b)(0, c)=(0,0)
$$

for any $c \in B$. By $b \in \mathrm{Ann}_{B} B$ it follows $z c=0$ for any $c \in B$, implying $z B=0$ and so also $z A=0$.

In analogy of the definition of the characteristic of a ring we introduce the notion of the characteristic of a class $\mathbf{C}$ defined by

$$
\operatorname{char} \mathbf{C}=\left\{\begin{array}{l}
\min (n: \forall A \in \mathbf{C}, n A=0 \text { for some } n=1,2, \ldots) \text { if exists, } \\
0 \text { otherwise. }
\end{array}\right.
$$

By definition it is clear that if $\operatorname{char} A=k$, $\operatorname{char} \mathbf{C}=n$ and $A \in \mathbf{C}$, then $k$ divides $n$.

Proposition 3. If $\mathbf{R}$ is a Dorroh essentially closed radical such that $Z \notin \mathbf{R}$ then $\operatorname{char} \mathbf{R} \neq 0$.

Proof. Since $Z \notin \mathbf{R}$, by Proposition 2 char $A \neq 0$ for all $A \in \mathbf{R}$. Let us consider the set

$$
\mathscr{P}=\{n: n=\operatorname{char} A \text { for some } A \in \mathbf{R}\} .
$$

We show that $\mathscr{P}$ is a finite set. Suppose that $\mathscr{P}$ is infinite. Since $\mathbf{R}$ is Dorroh essentially closed, to any $n \in \mathcal{P}$ there exists a ring $A_{n}$ such that $1 \in A_{n} \in \mathbf{R}$ and char $A_{n}=n$. Let us consider the direct sum $B=\sum_{n \in \mathcal{P}} A_{n}$, clearly $\operatorname{Ann}_{B} B=0$. For any ideal $I$ of $D(B)(D(B)=D(Z, B))$ with $I \cap B=0$ Lemma 2 gives

$$
I \subseteq\left\{(z, 0): z \in \mathrm{Ann}_{Z} B\right\} .
$$

If $0 \neq(z, 0 \in I)$, then as $\mathscr{P}$ is an infinite set, there exists an $n(n>z)$ such that $0 \neq z A_{n} \subseteq z B=0$, contradicting $Z \notin \mathbf{R}$. Thus $\mathscr{P}$ is a finite set. Hence char $\mathbf{R} \neq 0$.

Proposition 4. If $\mathbf{R}$ is a Dorroh essentially closed radical and $Z \notin \mathbf{R}$, then $Z /(\operatorname{char} \mathbf{R}) \in \mathbf{R}$. Moreover, for every $A \in \mathbf{R}: \operatorname{Ann}_{A} A=0$. 
Proof. By Proposition 3 we have char $\mathbf{R} \neq 0$. Since $\mathbf{R}$ is obviously closed under direct sums, there exists a $\operatorname{ring} A$ in $\mathbf{R}$, such that char $A=$ char $\mathbf{R}$. Without loss of generality we may assume that $1 \in A$ as $\mathbf{R}$ is Dorroh essentially closed. Let us consider the direct sum $B=\sum_{i=1}^{\infty}\left(A_{i}: A_{i} \cong A\right)$, and apply Lemma 2 for an ideal $I$ of $D(B)$ with $I \cap B=0$. As $\operatorname{Ann}_{B} B=0$, we get

$$
I \subseteq\left\{(z, 0): z \in \operatorname{Ann}_{Z} B\right\} .
$$

For any $(z, 0) \in I$ the characteristic $n$ of $\mathbf{R}$ divides $z$, hence $\left\{(z, 0): z \in \operatorname{Ann}_{Z} B\right\}$ equals to the ideal $J$ generated by $(n, 0)$. Thus $J \cap B=0$ and by $\operatorname{Ann}_{B} B=0, J$ is maximal with respect to this property. Since $D(B) / J \cong D(Z /(n), B)$, and hence it follows $D(Z /(n), B) \in \mathbf{R}$ and also

$$
Z /(n) \cong D(Z /(n), B) / B \in \mathbf{R} \text {. }
$$

Thus $Z /(n) \in \mathbf{R}$.

Next assume that $A \in \mathbf{R}$ and $\mathrm{Ann}_{A} A \neq 0$. Let $a \neq 0$ be in $\operatorname{Ann}_{A} A$, then the ideal $I$ generated by $\left(n^{2}, a\right)$ in ring $D(A)$ satisfies that $I \cap A=0$. Let $J$ be an ideal containing $I$, which is maximal with respect to $J \cap A=0$. Then by Lemma $1 J$ is isomorphic to an ideal of $Z$. Hence $J$ is generated by a single element $(z, b)$ such that $k(z, b)=\left(n^{2}, a\right)$ for some integer $k$. It is known that $Z /(z) \cong$ $D(A) /(J+A) \in \mathbf{R}$. This implies that $z$ divides $n$. On the other hand $k z=n^{2}$ and $k b=a$, hence $n$ divides $k$ and consequently $a=k b=0$, a contradiction. Thus $\operatorname{Ann}_{A} A=0$.

A characterization of Dorroh essentially closed radical classes not containing the ring $Z$ of integers is given in the following

THEOREM 2. Let $\mathbf{R}$ be non-empty class of rings. The following conditions are equivalent:

i) $\mathbf{R}$ is a Dorroh essentially closed radical and $Z \notin \mathbf{R}$,

ii) $\mathbf{R}$ is a radical class of nonzero characteristic and

a) $Z /(n) \in \mathbf{R}$, where $n=\operatorname{char} \mathbf{R}$,

b) $\operatorname{Ann}_{A} A=0$ holds for any $A \in \mathbf{R}$.

Proof. (i) implies (ii) from Proposition 3 and 4.

(ii) $\Rightarrow$ (i). Because $\mathbf{R}$ is a radical class, we have only to show that $Z \notin \mathbf{R}$ and $\mathbf{R}$ is Dorroh essentially closed. Since char $\mathbf{R} \neq 0$ it follows $Z \notin \mathbf{R}$. To prove that $\mathbf{R}$ is Dorroh essentially closed let $A \in \mathbf{R}$ and $J$ be an ideal of $D(A)$, which is maximal relative to $J \cap A=0$. By Lemma $1 J$ is generated by a single element $(z, a)$. Let us introduce the following notations: $m=\operatorname{char} A$ and

$$
\begin{gathered}
m=p_{1}^{\alpha_{1}} p_{2}^{\alpha_{2}} \cdots p_{r}^{\alpha_{r}}, \quad \text { o } a=p_{1}^{\beta_{1}} p_{2}^{\beta_{2}} \cdots p_{r}^{\beta_{r}}, \\
z=p_{1}^{\gamma_{1}} p_{2}^{\gamma_{2}} \cdots p_{r}^{\gamma_{r}} \cdot q \quad \text { where }\langle q, m\rangle=1 .
\end{gathered}
$$


Since $J \cap A=0$, for every $x \in A$ we have $z a+a x=0=z x+x a$ and hence $(o(a), z) x=0$. Thus $m$ divides $o(a) z$ and consequently $\alpha_{i} \leqslant \beta_{i}+\gamma_{i}$ for each $i=1,2, \ldots, r$. Let

$$
k=p_{1}^{\left(\beta_{1}+\gamma_{1}\right)-\alpha_{1}} \cdots p_{r}^{\left(\beta_{r}+\gamma_{r}\right)-\alpha_{r}}
$$

we shall show that $\langle\mathrm{o}(a), k\rangle=1$.

If $a=0$, then $\mathrm{o}(a)=1$, hence $\langle\mathrm{o}(a), k\rangle=1$.

Suppose that $a \neq 0$. Obviously $A$ is the direct sum of its $p$-components $\left(m / p_{i}^{\alpha_{i}}\right) A, i=1,2, \ldots, r$, and hence the element $a$ has the form $a=a_{1}+a_{2}$ $+\cdots+a_{r}, a_{i} \in m / p_{i}^{\alpha_{i}} A, i=1,2, \ldots, r$. Since $a \neq 0$, there exists an index $i$ such that $a_{i} \neq 0$. In this case $\beta_{i} \neq 0$ and o $\left(a_{i}\right)=p_{i}^{\beta_{1}}$. We shall show that $\alpha_{i}=\beta_{i}+\gamma_{i}$. Assume that $\alpha \subsetneq \beta_{i}+\gamma_{i}$, then by $(z, a) \in J$ it follows that the element $\left(z\left(\mathrm{o}(a) / p_{i}^{\beta_{i}}\right),\left(\mathrm{o}(a) / p_{i}^{\beta_{i}}\right) a\right)$ of $D(A)$ is in the ideal $J$. Consequently, $\left(z \cdot\left(\mathrm{o}(a) / p_{i}\right),\left(\mathrm{o}(a) / p_{i}\right) a_{i}\right) \in J$ also holds. Since $\alpha_{i} \Varangle \beta_{i}+\gamma_{i}$, it implies that $m$ divides $z \cdot\left(\mathrm{o}(a) / p_{i}\right)$. By $\left(z \cdot(\mathrm{o}(a) / p),\left(\mathrm{o}(a) / p_{i}\right) a_{i}\right) \in J$ we have that for all $x \in A$

$$
\begin{aligned}
& 0=z \cdot \frac{\mathrm{o}(a)}{p_{i}} x+\frac{\mathrm{o}(a)}{p_{i}} x a_{i}=\frac{\mathrm{o}(a)}{p_{i}} x a_{i}, \\
& 0=z \cdot \frac{\mathrm{o}(a)}{p_{i}} x+\frac{\mathrm{o}(a)}{p_{i}} a_{i} x=\frac{\mathrm{o}(a)}{p_{i}} a_{i} x .
\end{aligned}
$$

Hence $\left(\mathrm{o}(a) / p_{i}\right) x a_{i}=\left(\mathrm{o}(a) / p_{i}\right) a_{i} x$ for all $x \in A$. This means that $\left(\mathrm{o}(a) / p_{i}\right) a_{i} \in$ $\operatorname{Ann}_{A} A=0$. Thus $\left(\mathrm{o}(a) / p_{i}\right) a_{i}=0$. But $\mathrm{o}\left(a_{i}\right)=p_{i}^{\beta_{i}}$, a contradiction. Hence $\alpha_{i}=\beta_{i}+\gamma_{i}$ for all $a_{i} \neq 0$.

If $a_{j}=0$ then $\mathrm{o}\left(a_{j}\right)=1$, thus $\beta_{j}=0$, and hence $\left\langle p_{j}, \mathrm{o}(a)\right\rangle=1$. This reasoning has proved $\langle k, \mathrm{o}(a)\rangle=1$. By $\langle k, \mathrm{o}(a)\rangle=1$ there exists an element $c \in A$ such that $o(a)=\mathrm{o}(c)$ and $a=k c$. Let us consider the ideal $I$ generated by the element $(z / k, c)$ then for any element $y \in A$

$$
k\left(\frac{z}{k} y+y c\right)=z y+y(k c)=z y+y a=0
$$

and similarly $k((z / k) y+c y)=0$. On the other hand

$$
\mathrm{o}(a)\left(\frac{z}{k} y+y c\right)=\frac{\mathrm{o}(a)}{k} z y+y(\mathrm{o}(a) c)=0
$$

is valid which implies that if $((z / k) y+y c) \neq 0$ then $\mathrm{o}((z / k) y+y c)$ divides $m$. By $\langle k, o(a)\rangle=1$ and $k((z / k) y+y c)=0$ we get that $(z / k) y+y c=0=$ $(z / k) y+c y$. Hence the intersection of $I$ with $A$ is zero. Since $I$ contains $J$, by the maximality of $J$ it follows that $J=I$. Hence $k=1$ and $c=a$ hold. It implies that $q=1$ and $\alpha_{i}=\beta_{i}+\gamma_{i}$ for all $i=1,2, \ldots, r$. Moreover, $z$ divides $m$ and also $n=$ char $\mathbf{R}$. By condition (a), $Z /(n) \in \mathbf{R}$ implies $Z /(z) \in \mathbf{R}$. We have now 
$D(A) /(J+A) \cong Z /(z) \in \mathbf{R}$. Furthermore as $(J+A) / J \cong A$ and

$$
\frac{D(A) / J}{(J+A) / J} \cong Z /(z) \in \mathbf{R}
$$

the extension property of $\mathbf{R}$ yields $D(A) / J \in \mathbf{R}$; hence $\mathbf{R}$ is Dorroh essentially closed.

CorollaRY. If $\mathbf{R}$ is a Dorroh essentially closed radical and $Z \notin \mathbf{R}$, then $\mathbf{R}$ is a weakly subidempotent radical. Moreover, if $A \in \mathbf{R}$, then $a \in A a+a A+A a A$ holds for all $a \in A$.

Proof. Taking into consideration that $A \in \mathbf{R}$ implies $A / A^{2} \in \mathbf{R}$ and (b) it follows that $A / A^{2}=0$. Hence $\mathbf{R}$ consists of idempotent rings.

Since the element $a+(A a+a A+A a A) \in A /(A a+a A+A a A)=B$ is in the annihilator of $B$, condition (b) yields the second assertion.

Let us consider the class

$$
\mathbf{B}=\left\{A: \operatorname{Ann}_{B} B=0 \text { for every homomorphic image } B \text { of } A\right\} .
$$

This class of rings has been introduced by F. A. Szász (and denoted by $\mathbf{B}_{6}$ ) and it has been proved in [5] that $\mathbf{B}$ is a non-hereditary radical class consisting of idempotent rings. Further, let $\mathbf{T}_{n}$ denote the class $\mathbf{T}_{n}=\{A: n A=0\}$ for any $n>0$.

Proposition 5. The class $\mathbf{B}_{n}=\mathbf{B} \cap \mathbf{T}_{n}$ is the largest Dorroh essentially closed radical of characteristic $n$.

Proof. Obviously $\mathbf{B}_{n}$ is homomorphically closed and has the inductive property. (The class $\mathbf{C}$ has the inductive property, if for any ascending chain $I_{1} \subseteq$ $\cdots \subseteq I_{\gamma} \subseteq \cdots$ of ideals of a ring $A$ such that $I_{\gamma} \in \mathbf{C}$ for each $\gamma$, it follows $\cup_{\gamma} I_{\gamma} \in \mathbf{C}$.) Next, let $A \triangleleft B$ such that $A, B / A \in \mathbf{B}_{n}$. Since $\mathbf{B}$ is a radical class, it suffices to show that $n B=0$. As $B / A \in \mathbf{T}_{n}$, we have $n B \subseteq A$, implying $n B A=$ $B n A=0$. Thus $n B \subseteq \operatorname{Ann}_{A} A=0$ holds as $A \in \mathbf{B}_{n}$, and hence $n B=0$. Thus $\mathbf{B}_{n}$ is a radical class. Applying Theorem $2 \mathbf{B}_{n}$ is a Dorroh essentially closed radical with $Z \notin \mathbf{B}_{n}$. By the definition of $\mathbf{B}_{n}$ and by Theorem 2 the maximality of $\mathbf{B}_{n}$ is obvious.

Next we turn our attention to Dorroh essentially closed radicals, which are hereditary. 
THEOREM 3. Let $\mathbf{R}$ be a hereditary radical. The following two conditions are equivalent:

i) $\mathbf{R}$ is Dorroh essentially closed and $Z \notin \mathbf{R}$,

ii) $\mathbf{R}$ is subidempotent of nonzero characteristic $n$ and $Z /(n) \in \mathbf{R}$.

Moreover, $n=$ char $\mathbf{R}$ is a square-free integer.

Proof. By the corollary of Theorem $2 \mathbf{R}$ is subidempotent, moreover, by Theorem $2 \mathbf{R}$ satisfies the further conditions.

Conversely, as $\mathbf{R}$ is hereditary, condition (b) of Theorem 2 is satisfied and hence by Theorem $2 \mathbf{R}$ is Dorroh essentially closed and also $Z \notin \mathbf{R}$ holds.

It is well-known that if $A$ is a hereditarily idempotent ring of nonzero characteristic $n$, then $n$ is a square-free integer. This proves the second assertion of the theorem.

Summarizing our results concerning essentially closed, Dorroh essentially closed and Dorroh essentially closed hereditary radicals, respectively, we obtain the following description of such radicals. We say that a class $\mathbf{C}$ of rings is the direct sum $\mathbf{C}=\mathbf{C}_{1} \oplus \cdots \oplus \mathbf{C}_{r}$ of subclasses $\mathbf{C}_{i}, i=1,2, \ldots, r$, if $A=A_{1} \oplus A_{2} \oplus \cdots \oplus A_{r}$, $A_{i} \in \mathbf{C}_{i}, i=1, \ldots, r$, holds for each $A \in \mathbf{C}$.

THEOREM 4. Let $\mathbf{R}$ be any radical with $Z \notin \mathbf{R}$.

1) If $\mathbf{R}$ is Dorroh essentially closed, then

$$
\mathbf{R}=\mathbf{R}_{1} \oplus \mathbf{R}_{2} \oplus \cdots \oplus \mathbf{R}_{r}
$$

where each $\mathbf{R}_{t}=\mathbf{R} \cap \mathbf{B}_{p^{\alpha_{i}}}$ is a Dorroh essentially closed radical and char $\mathbf{R}=$ $\Pi_{i=1}^{r} p_{i}^{\alpha_{i}}$. Conversely, if $\mathbf{R}_{i}, i=1, \ldots, r$, are Dorroh essentially closed radicals such that char $\mathbf{R}_{i}=p_{i}^{\alpha_{i}}, p_{i} \neq p_{j}$ for $i \neq j$ (every $p_{i}$ is prime), then also their direct sum is a Dorroh essentially closed radical.

2) If $\mathbf{R}$ is in addition hereditary, then $\alpha_{i}=1$ for each $i=1, \ldots, r$ and each $\mathbf{R}_{i}$ is hereditary. Also the converse implication holds.

3) If $\mathbf{R}$ is any essentially closed radical, then $\mathbf{R}$ is a direct sum

$$
\mathbf{R}=\mathbf{R}_{1} \oplus \mathbf{R}_{2} \oplus \cdots \oplus \mathbf{R}_{r}
$$

where char $\mathbf{R}_{i}=p_{i}, i=1, \ldots, r, p_{i} \neq p_{j}$ for $i \neq j$ and for each $i=1, \ldots, r$ there is $a$ strictly hereditary finite set $\mathbf{F}_{i}$ of finite fields such that each ring $A$ of $\mathbf{R}_{i}$ is a subdirect sum of fields from $\mathbf{F}_{i}$. The converse statement is also true.

Proof. The assertion of (1) and (2) are straightforward in view of our proved results, further (3) is Stewart's [4] Theorem 4.3. 
Let us remark that, obviously there exist radicals satisfying the conditions of (1) but not those of (2) and radicals satisfying the conditions of (2) but not those of (3) respectively.

\section{References}

[1] E. P. Armendariz, 'Closure properties in radical theory', Pacific J. Math. 26 (1968) 1-7.

[2] B. J. Gardner and P. N. Stewart, 'On semisimple radical classes', Bull. Austral. Math. Soc. 13 (1975) 349-353.

[3] L. C. A. van Leeuwen, 'Properties of semisimple classes', J. Natur. Sci. and Math. 15 (1975) 59-67.

[4] P. N. Stewart, 'Semisimple radical classes', Pacific J. Math. 32 (1970) 249-254.

[5] F. A. Szász, 'A second almost subidempotent radical for rings', Math. Nachr. 66 (1975) 283-289.

[6] R. Wiegandt, Radical and semisimple classes of rings (Queen's Papers in Pure and Appl. Math. 37 Kingston 1974).

\section{Eötvös University}

Budapest

Hungary 